\title{
Approaches in Sepsis Prevention
}

\section{Rabie MA*}

Faculty of Pharmacy, Cairo University, Egypt

*Corresponding author: Rabie MA, Faculty of Pharmacy, Cairo University, Egypt,

\section{Mini Review}

Volume 2 Issue 1

Received Date: April 06, 2017

Published Date: May 02, 2017

Tel: 201224247229; E-mail: maha.ahmed@pharma.cu.edu.eg

\section{Abstract}

Background: Lipopolysaccharide (LPS) is recognized by the innate immune system via toll-like receptor 4 (TLR4) whose activation leads to the production of numerous immunoregulatory molecules and reactive oxygen species.

Aim: This study was designed to investigate the effects of different interventions on LPS-induced renal oxidative and immunological changes of mice.

Methods: Male Swiss mice were injected with LPS (1 mg/kg; i.p.) and pretreatment with baicalin (50 mg/kg; i.p.), parthenolide (10 mg/kg/day, i.p), SC-58125 (10 mg/kg/day, i.p) as well as ZVAD (2 mg/kg/day, i.p) of LPS-induced renal failure and kidney pathology 3 or 24 h post LPS injection. Kidney content of interleukin-1 $\beta$ (IL-1 $\beta$ ) and IL-10 were assessed. Oxidative stress as well as the RNA expression of neutrophil gelatinase-associated lipocalin (NGAL) and inhibitor of nuclear factor-kappa B (NF- $\mathrm{kB})$ alpha $(\mathrm{I} \kappa \mathrm{B} \alpha)$ in the kidney were also evaluated.

Results: LPS augmented renal malondialdehyde and IL-10 levels as well as caspase-3 activity. However, it diminished the reduced glutathione and IL-1 $\beta$ levels; besides, it inhibited superoxide dismutase and catalase activities in the kidney. Histopathologic studies backed the previous observations. The studied agents significantly ameliorated LPS-induced alterations and suppressed acute kidney injury (AKI) by modulating NGAL and I BB- $\alpha$ mRNA levels. In conclusion, the present study suggested that the used drugs had potential beneficial role in sepsis prevention and its associated renal derangements.

Keywords: Gene Expression; Interleukins; LPS; Oxidative Stress

\section{Approaches in Sepsis Prevention}

Sepsis is a systemic inflammatory response syndrome that can lead to lethal organ damage. Despite advances in modern intensive care, the overall mortality of severe sepsis exceeds $30 \%$. It is the third leading cause of death after cardiovascular disease and cancer. Death from severe sepsis occurs because of multi-organ failure, especially the lung, liver, and kidney [1]. Lipopolysaccharide (LPS) induces systemic inflammation that mimics many early clinical features of sepsis. Its exposure also causes renal injury and microcirculatory failure [2]. So far, there has been no effective treatment for sepsis-induced acute kidney injury (AKI). Therefore, novel therapeutic interventions are urgently needed to tackle this distressing disease.

The present experiments were constructed on male albino wild-type Swiss mice, weighing 20-30 g) to investigate the possible mechanism(s) involved in AKI 


\section{Advances in Pharmacology and Clinical Trials}

from LPS administration ( $1 \mathrm{mg} / \mathrm{kg}$; i.p.) and the effect of different therapeutic strategies by the use of baicalin (50 $\mathrm{mg} / \mathrm{kg}$; i.p.) as toll like receptor (TLR) 4 inhibitor, parthenolide $(10 \mathrm{mg} / \mathrm{kg} /$ day, i.p) as anti-inflammatory, SC-58125 (10 mg/kg/day, i.p) as cyclooxygenase (COX)-2 inhibitor as well as ZVAD $(2 \mathrm{mg} / \mathrm{kg} /$ day, i.p) as caspase inhibitor at both $3 \mathrm{~h}$ - and $24 \mathrm{~h}$ - phases. Each drug was administered in three successive doses at 48,24 and $3 \mathrm{~h}$ before LPS injection. Oxidative stress, as well as the RNA expression of high-mobility group box1 (HMGB1) and

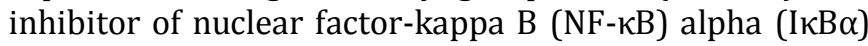
in the kidney, were evaluated. The study was performed according to the guidelines stated by the ethics committee (approval no. PT 596).

Inflammation in sepsis is largely initiated by TLRs, which detect not only a wide range of microbial diversity, but also cellular constituents released due to tissue injury as well as triggering innate immune responses [3]. Baicalin, a flavonoid compound of plant origin, has been shown to be a potent antagonist of the biochemical and physiological effects of LPS and immune regulation through inhibition of TLR4. Baicalin possesses antiinfective, anti-inflammatory as well as antioxidant effects [4,5]. HMGB1 can potentiate TLR ligand signals [6] and perhaps even act as a TLR ligand [7].

Nuclear factor- $\kappa \mathrm{B}(\mathrm{NF}-\kappa \mathrm{B})$ has long been considered a prototypical proinflammatory signaling pathway in the expression of other proinflammatory genes including cytokines, chemokines and adhesion molecules [8]. A proposed parthenolide mechanism of action involves inhibition of the NF- $\kappa B$ pathway by blocking IKK- $\beta$, an activator of NF- $\kappa B$ [9]. The IKK- $\beta$ complex plays an important role in pro-inflammatory cytokine-mediated signaling. Lipopolysaccharide (LPS) is known to cause an increase in markers of oxidative stress [10] and an upregulation of renal Fas and Fas-ligand expression [11] thus activating both intrinsic and extrinsic apoptotic pathways.

Among the multiple mediators of the complex process of apoptosis, the caspase enzyme cascade plays a central role in the production of several inflammatory cytokines, and apoptosis [12]. The efficacy of potent peptide based macromolecular inhibitors such as Z-VAD in clinical models of disease may be a consequence of their ability to inhibit multiple caspases [13].

Cyclooxygenase (COX)-2, has been identified to be highly expressed in inflammatory tissues [14] where it produces pro-inflammatory prostaglandin (PG). The gene for COX-2 has NF- $\kappa B$ binding sequences in its promoter region, which are critical for transcriptional activation [15]. Thus, enhanced COX-2 activity induced by LPS is due to the selective increase in levels of COX-2 protein, suggesting that up-regulation of COX-2 is responsible for increased synthesis of PG and thromboxanes [16].

The present work revealed that LPS, in the used dose $(1 \mathrm{mg} / \mathrm{kg})$, had preconditioning effect as manifested by elevation of anti-inflammatory interleukin (IL)-10, suppression of pro-inflammatory IL-1 $\beta$, enhancing gene expression of inhibitor of nuclear factor-kappa B (NF-kB)

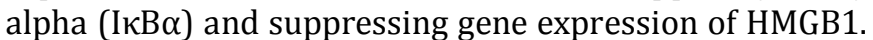
This preconditioning was more predominant at early phase than in the late phase. Neither oxidative stress nor apoptotic pathways was included in this preconditioning. The effect of preconditioning did not extend to 24-h phase and this could explain how death occurred due to LPS administration.

In the current investigation, pretreatment with baicalin, parthenolide, SC-58125 or Z-VAD attenuated the LPS-induced AKI as manifested by attenuated kidney functional impairment, counteracted the state of oxidative stress, further enhanced preconditioning effects of LPS, ameliorated apoptotic cell death as well as improved kidney histopathologic changes. These tested agents possibly exerted their nephroprotective effects via their antioxidant and/or anti-inflammatory properties. Histopathologic studies backed the previous observations.

The most promising drugs were parthenolide, SC58125 and Z-VAD where they effectively enhanced survival percentage at $24 \mathrm{~h}$-phase. The advantage of baicalin and parthenolide over other interventions is that they are originated from plant source, so adverse effects could be reduced. The recommended future study can be designed to shed some light on whether the combination of different drugs with different mechanisms could offer a promising effect against LPS-induced AKI and further increase survival percentage or even prevent death due to Gram negative bacteria especially in intensive care units.

\section{References}

1. Dellinger RP, Levy MM, Carlet JM, Bion J, Parker MM, et al. (2008) Surviving Sepsis Campaign: international guidelines for management of severe sepsis and septic shock. Crit Care Med 36: 296-327. 


\section{Advances in Pharmacology and Clinical Trials}

2. Doi K, Leelahavanichkul A, Yuen P, Star R (2009) Animal models of sepsis and sepsis-induced kidney injury. J Clin Invest 119(10): 2868-2878.

3. Medzhitov R (2007) Recognition of microorganisms and activation of the immune response. Nature 449(7164): 819-826.

4. Gao Z, Huang K, Xu H (2001) Protective effects of flavonoids in the roots of Scutellaria baicalensis Georgi against hydrogen peroxide-induced oxidative stress in HS-SY5Y cells. Pharmacol Res 43(2): 173178.

5. Ouyang C, Wu J (2006) Protective effect of baicalin on inflammatory injury following transient focal cerebral ischemia reperfusion in rats. Chinese J Pharmacol Toxicol 20(4): 288-294.

6. Tian J, Avalos AM, Mao SY, Chen B, Senthil K, et al. (2007) Toll-like receptor 9-dependent activation by DNA-containing immune complexes is mediated by HMGB1 and RAGE. Nat Immunol 8(5): 487-496.

7. van Zoelen MA, Yang H, Florquin S, Meijers JC, Akira S, et al. (2009) Role of toll-like receptors 2 and 4, and the receptor for advanced glycation end products in high-mobility group box 1-induced inflammation in vivo. Shock 31(3): 280-284.

8. Lawrence $\mathrm{T}$ (2009) The nuclear factor NF- $\kappa \mathrm{B}$ pathway in inflammation. Cold Spring Harb Perspect Biol 1(6): 1-10.

9. Sur R, Martin K, Liebel F, Lyte P, Shapiro S, et al. (2009) Anti-inflammatory activity of parthenolidedepleted feverfew (Tanacetum parthenium). Inflammopharmacol 17(1): 42-49.
10. Wiesel P, Patel AP, DiFonzo N, Marria PB, Sim CU, et al. (2000) Endotoxin-induced mortality is related to increased oxidative stress and end-organ dysfunction, not refractory hypotension, in heme oxygenase-1deficient mice. Circulation 102(24): 3015-3022.

11. Koide N, Narita K, Kato Y, Sugiyama T, Chakravortty D, et al. (1999) Expression of Fas and Fas ligand on mouse renal tubular epithelial cells in the generalized Shwartzman reaction and its relationship to apoptosis. Infect Immun 67(8): 4112-4118.

12. Los M, Wesselborg S, Schulze-Osthoff K (1999) The role of caspases in development, immunity, and apoptotic signal transduction: Lessons from knockout mice. Immunity 10(6): 629-639.

13. Garcia-Calvo M, Peterson E, Leiting B, Ruel R, Nicholsoni DW, et al. (1998) Inhibition of human caspases by peptide-based and macromolecular inhibitors. J Biol Chem 273(49): 32608-32613.

14. Hla T, Neilson K (1992) Human cyclooxygenase-2 cDNA. Proc Natl Acad Sci 89(16): 7384-7388.

15. Schmedtje JF Jr, Ji YS, Liu WL, DuBois RN, Runge MS (1997) Hypoxia induces cyclooxygenase-2 via the NFkappaB p65 transcription factor in human vascular endothelial cells. J Biol Chem 272(1): 601-608.

16. Phillips TA, Kujubu DA, MacKay RJ, Herschman HR, Russell SW, et al. (1993) The mouse macrophage activation-associated marker protein, $\mathrm{p} 71 / 73$, is an inducible prostaglandin endoperoxide synthase (cyclooxygenase). J Leukoc Biol 53(4): 411-419. 